Georgian Mathematical Journal

Volume 13 (2006), Number 4, 783-786

\title{
SOME PROPERTIES OF THE INVERSION COUNTING FUNCTION
}

\author{
NEVILLE ROBBINS
}

\begin{abstract}
Let $h, k$ be integers such that $0<h<k$ and $(h, k)=1$. If $1 \leq i \leq k-1$, let $r_{i}$ be the least positive residue $(\bmod k)$ of $h i$. Let the permutation

$$
\sigma_{h, k}=\left(\begin{array}{rrrrr}
1 & 2 & 3 & \cdots & k-1 \\
r_{1} & r_{2} & r_{3} & \cdots & r_{k-1}
\end{array}\right)
$$

For $1 \leq i<j \leq k-1$, if $r_{i}>r_{j}$, this is called an inversion of $\sigma_{h, k}$. Let $I(h, k)$ denote the total number of inversions of $\sigma_{h, k}$. In this note, we prove several identities concerning $I(h, k)$.
\end{abstract}

2000 Mathematics Subject Classification: 11A25.

Key words and phrases: Inversions, Dedeking sums.

1. Introduction. Let $h, k$ be integers such that $0<h<k$ and $(h, k)=1$. Consider the permutation

$$
\sigma_{h, k}=\left(\begin{array}{rrrrr}
1 & 2 & 3 & \cdots & k-1 \\
r_{1} & r_{2} & r_{3} & \cdots & r_{k-1}
\end{array}\right)
$$

where $r_{i}$ is the least positive residue $(\bmod k)$ of $h i$ for $1 \leq i \leq k-1$. In particular, if $p$ is an odd prime and if $0<h<p$, then the Legendre symbol $\left(\frac{h}{p}\right)=1$ if and only if $\sigma_{h, p}$ is the product of an even number of transpositions. (See [2], p. 280.) Let $I_{i}(h, k)$ denote the number of elements in the sequence $\left\{r_{1}, r_{2}, r_{3}, \ldots, r_{i-1}\right\}$ that exceed $r_{i}$. Let

$$
I(h, k)=\sum_{i=1}^{k-1} I_{i}(h, k) .
$$

Note that $I(h, k)$ counts the number of so-called inversions in $\sigma_{h, k}$. (The term inversion is due to C. Meyer [3].) In [4], several identities concerning $I(h, k)$ were presented. In this note, we develop further properties of $I(h, k)$.

2. Preliminaries. Let $s(h, k)$ denote the usual Dedekind sum.

$$
\begin{gathered}
I(h, k)=-3 k s(k, h)+\frac{1}{4}(k-1)(k-2) . \\
h I(h, k)+k I(k, h)=\frac{1}{4}(h-1)(k-1)(h+k-1) . \\
\text { If } h^{\prime} \equiv \pm h(\bmod k), \text { then } s\left(h^{\prime}, k\right)= \pm s(h, k) . \\
\text { If } h^{\prime} \equiv h(\bmod k), \quad \text { then } I\left(h^{\prime}, k\right)=I(h, k) .
\end{gathered}
$$


Remarks. (1), (2) are (48), (50) respectively in [4]. (See p. 37, 39.) (2) is attributed by Meyer to H. Salie. (See [3], p. 163.) (3) is Theorem 3.6, part (a) on p. 62 of [1]. (4) follows from (1) and (3). An additional identity ((49) in [4]) states that

$$
(-1)^{I(h, k)}=\left(\frac{h}{k}\right)
$$

This last identity, sometimes called Zolotarev's Theorem, yields an alternate proof of the quadratic reciprocity law for the Jacobi symbol.

\section{The Main Results.}

Theorem 1. Let $h, k$ be integers such that $0<h<k$ and $(h, k)=1$. Then

$$
\begin{aligned}
& \text { (a) } I(k-h, k)+I(h, k)=\frac{(k-1)(k-2)}{2}, \\
& \text { (b) } I(k-h, k)-I(h, k)=6 k s(h, k) .
\end{aligned}
$$

Proof. Since (3) implies $s(k-h, k)=s(-h, k)=-s(h, k)$, it follows from (1) that

$$
I(k-h, k)=3 k s(h, k)+\frac{1}{4}(k-1)(k-2) .
$$

The conclusion now follows from (1) and (7).

Theorem 2. If $k$ is odd, then

$$
\sum_{h=1}^{k-1} I(h, k)=\frac{1}{4}(k-1)^{2}(k-2) .
$$

Proof.

$$
\sum_{h=1}^{k-1} I(h, k)=\sum_{h=1}^{\frac{k-1}{2}} I(h, k)+\sum_{h=\frac{k+1}{2}}^{k-1} I(h, k)
$$

but

$$
\sum_{h=\frac{k+1}{2}}^{k-1} I(h, k)=\sum_{h=1}^{\frac{k-1}{2}} I(k-h, k)
$$

so that, using Theorem 1(a), we get:

$$
\begin{aligned}
\sum_{h=1}^{k-1} I(h, k)=\sum_{h=1}^{\frac{k-1}{2}}(I(h, k) & +I(k-h, k)) \\
= & \sum_{h=1}^{\frac{k-1}{2}} \frac{1}{2}(k-1)(k-2)=\frac{1}{4}(k-1)^{2}(k-2) .
\end{aligned}
$$

Theorem 3. If $0<h<k,(h, k)=1$ and $k \equiv 0(\bmod 3)$, then $I(h, k) \equiv$ $h-1(\bmod 3)$. 
Proof. By hypothesis, we must have $h \not \equiv 0(\bmod 3)$. Therefore, by $(2)$ and the hypothesis, we have

$$
h I(h, k) \equiv-(h-1)^{2} \quad(\bmod 3)
$$

hence

$$
I(h, k) \equiv-h+2-h^{-1} \quad(\bmod 3)
$$

from which the conclusion follows.

Theorem 4. If $0<h<k,(h, k)=1$, and $k \not \equiv 0(\bmod 3)$, then $I(h, k) \equiv 0$ $(\bmod 3)$.

Proof (Induction on $k)$. Note that $I(1,2)=0 \equiv 0(\bmod 3)$. First assume that

(a) $h \not \equiv 0(\bmod 3)$.

Now (2) implies

$$
h I(h, k)+k I(k, h) \equiv 0 \quad(\bmod 3) .
$$

Let $k=q h+r$, where $0<r<h$, then $I(k, h)=I(r, h)$, so that

$$
h I(h, k)+k I(r, h) \equiv 0 \quad(\bmod 3) .
$$

By the induction hypothesis, we have $I(r, h) \equiv 0(\bmod 3)$. Therefore $I(h, k) \equiv$ $0(\bmod 3)$. Now assume that

(b) $h \equiv 0(\bmod 3)$, so that $k-h \not \equiv 0(\bmod 3)$.

Since, by hypothesis, $k \neq \equiv 0(\bmod 3)$ it follows that $3 \mid(k-1)(k-2)$. Therefore (2) implies

$$
I(h, k)+I(k-h, k) \equiv 0 \quad(\bmod 3) .
$$

Our result from part (a) implies $I(k-h, k) \equiv 0(\bmod 3)$ so that $(9)$ implies $I(h, k) \equiv 0(\bmod 3)$.

\section{Theorem 5.}

(a) $\quad I(m, m n \pm 1)=\frac{m(m-1) n(n \pm 1)}{4}$.

(b) If $m$ is odd, then $I(m, m n \pm 2)=\frac{n(m-1)(2 m n \pm m \pm 3)}{8}$.

Proof. These results are obtained via (2) and (4).

\section{REFERENCES}

1. T. M. Apostol, Modular functions and Dirichlet series in number theory. 2nd ed. Graduate Texts in Mathematics, 41. Springer-Verlag, New York, 1990.

2. P. Bachmann, Niedere Zahlentheorie, I. B. G. Teubner, Leipzig, 1902.

3. C. Meyer, Über einige Anwendungen Dedekindscher Summen. J. Reine Angew. Math. 198(1957), 143-203.

4. H. Rademacher and E. Grosswald, Dedekind sums. The Carus Mathematical Monographs, No. 16. The Mathematical Association of America, Washington, D.C., 1972. 
(Received 12.09.2006)

Author's address:

Mathematics Department

San Francisco State University

San Francisco, CA 94132

USA

E-mail: robbins@math.sfsu.edu 